\title{
Diplopia and Ptosis as the Initial Manifestations of Acquired Hepatocerebral Degeneration
}

\author{
Jiguo Gao, Xiaoke Wang, Wei Wu, Xiujuan Wu, Hongliang Zhang
}

Keywords: Clinical neurosciences, neuroimaging, neurotoxicity, NMR spectroscopy

doi:10.1017/cjn.2015.283

Can J Neurol Sci. 2015; 42: 444-445

\section{Case Presentation}

A 62-year-old woman presented with an 18-day history of diplopia and right-sided ptosis. She had been positive for hepatitis $\mathrm{C}$ virus for 10 years and had been diagnosed with hepatic cirrhosis 4 years before. The patient complained of limb weakness 2 years ago and was diagnosed with hepatic myelopathy. T1-weighted images (T1WI) of magnetic resonance imaging (MRI) showed hyperintensity primarily in the bilateral globus pallidus and cerebral peduncle. However, no focal neurological symptoms or signs were noted at that time. Intravenous dexamethasone pulse treatment resulted in complete resolution. Upon admission, neurological examination showed right-sided ptosis, anisocoria (left, $3.0 \mathrm{~mm}$; right, $3.5 \mathrm{~mm}$ ), and upward gaze diplopia. A hyperactive deep tendon reflex was noted in the lower limbs. The Hoffmann sign was positive on the left side and the Babinski sign was positive bilaterally. The pain and temperature sensation was absent below the C4 level. T1WI showed symmetric hyperintensity primarily in the globus pallidus and the internal capsule (Figure 1A) as well as the cerebral peduncle (Figure 1B) (blue arrows). The results of contrast-enhanced MRI, magnetic resonance spectroscopy, and cerebrospinal fluid examination were unremarkable. The neostigmine test was negative. The blood level of ammonia was $177 \mu \mathrm{mol} / \mathrm{L}$, albumin $22.3 \mathrm{~g} / \mathrm{L}$, cholinesterase $1926 \mathrm{U} / \mathrm{L}$, and fasting glucose within the normal range. Abdominal ultrasound revealed ascites. Albumin, vitamin B, reduced glutathione, and diammonium glycyrrhizinate did not improve the patient's symptoms. The history of liver disease and typical imaging findings pointed to a diagnosis of acquired hepatocerebral degeneration (AHD).

Acquired hepatocerebral degeneration is a progressive neurological syndrome caused by various progressive liver diseases, and is characterized by parkinsonism, ataxia, and other movement disorders. ${ }^{1}$ T1WI hyperintensity found in the globus pallidus of patients with AHD is due to manganese deposits. ${ }^{2}$ However, the exact mechanism of action of manganese neurotoxicity and the reason for the relatively selective vulnerability of the globus pallidus is not known. Oxidative stress has been proposed as an important mechanism mediating manganese toxicity. ${ }^{3,4}$ Although the clinical manifestations of AHD are heterogeneous, the most commonly reported are motor signs such as tremor, akinesia, choreoathetosis, myoclonus, dysarthria, ataxia, and pyramidal signs. ${ }^{5}$ In this case, involvement of the interpeduncular fossa and oculomotor nerves may explain the diplopia and ptosis, which has not been documented in literature. Extrapyramidal signs such as parkinsonism were absent in our patient. One explanation is that the damage from manganese deposition to the substantia nigra and the globus pallidus is limited. With the progression of the disease, extrapyramidal signs may gradually develop.

\section{Disclosures}

None.

\section{Statement of Authorship}

The study concept and design was developed by HZ. Data were acquired by JG, X. Wang, WW, and X. Wu. The manuscript was drafted by JG, X. Wang, and WW. JG, XW, and WW contributed equally to this work.

\section{REFERENCES}

1. Fernández-Rodriguez R, Contreras A, De Villoria JG, Grandas F. Acquired hepatocerebral degeneration: clinical characteristics and MRI findings. Eur J Neurol. 2010;17:1463-70.

2. Fabiani G, Rogacheski E, Wiederkehr JC, Khouri J, Cianfarano A. Liver transplantation in a patient with rapid onset parkinsonismdementia complex induced by manganism secondary to liver failure. Arq Neuroqsiquiatr. 2007;65:685-8.

3. HaMai D, Bondy SC. Oxidative basis of manganese neurotoxicity. Ann N Y Acad Sci. 2004;1012:129-41.

4. Cersosimo MG, Koller WC. The diagnosis of manganese-induced parkinsonism. Neurotoxicology. 2006;27:340-6.

5. Burkhard PR, Delavelle J, Du Pasquier R, Spahr L. Chronic parkinsonism associated with cirrhosis. A distinct subset of acquired hepatocerebral degeneration. Arch Neurol. 2003;60:521-8.

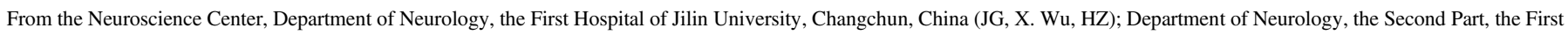

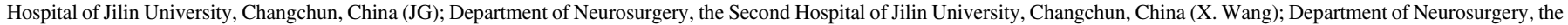
First Hospital of Jilin University, Changchun, China (WW)

Received SePtember 28, 2014. Final Revisions Submitted March 30, 2015

Correspondence to: Hongliang Zhang, Department of Neurology, the First Hospital of Jilin University, Jilin University, Xinmin Street 71\#, 130021, Changchun, China.

Email:drzhl@hotmail.com. 


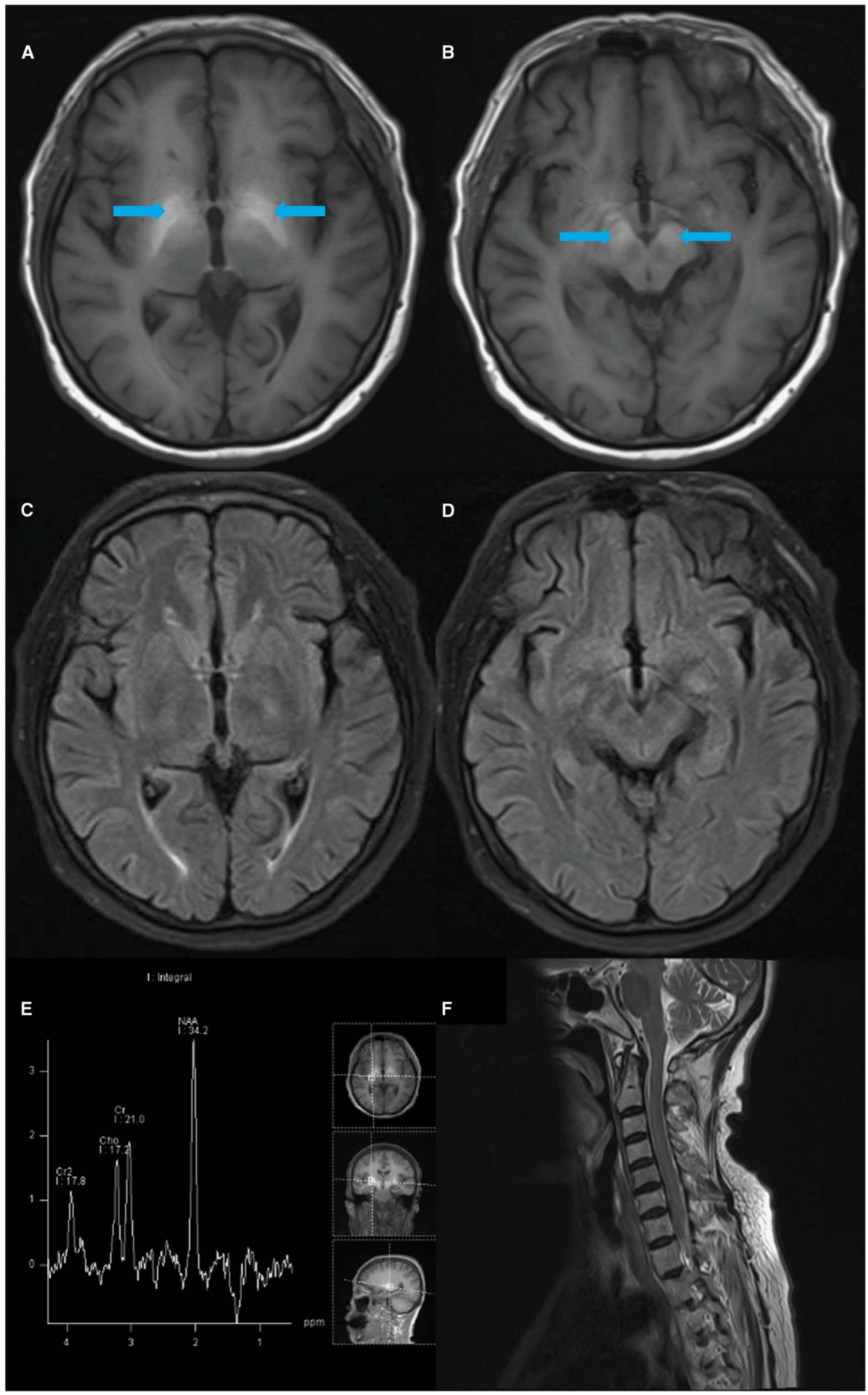

Figure 1: T1WI of an MRI showed symmetric hyperintensity primarily in the globus pallidus and the internal capsule (A) as well as the cerebral peduncle (B) (blue arrows). These anomalies are unremarkable in fluidattenuated inversion recovery $(C, D)$, magnetic resonance spectroscopy $(E)$ and MRI-T2-weighted images of the spinal cord $(F)$. The results of contrast-enhanced MRI were unremarkable (image not shown). 